\title{
Musical dual-task training in patients with mild-to- moderate dementia: a randomized controlled trial
}

This article was published in the following Dove Press journal:

Neuropsychiatric Disease and Treatment

\section{Yu-Ling Chen ${ }^{1,2}$ \\ Yu-Cheng $\mathrm{Pei}^{3-6}$}

'Department of Music, Southwestern Oklahoma State University,

Weatherford, OK, USA; ${ }^{2}$ Division of Music Education and Music Therapy, University of Kansas, Lawrence, KS, USA; ${ }^{3}$ Department of Physical Medicine and Rehabilitation, Chang Gung Memorial Hospital, Taoyuan, Taiwan; ${ }^{4}$ Center of Vascularized Tissue Allograft, Chang Gung Memorial Hospital at Linkou, Taoyuan, Taiwan; ${ }^{5}$ School of Medicine, Chang Gung University, Taoyuan, Taiwan; ${ }^{6}$ Healthy Aging Research Center, Chang Gung University, Taoyuan, Taiwan
Correspondence: Yu-Cheng Pei Department of Physical Medicine and Rehabilitation, Chang Gung Memorial Hospital, 5 Fu-shin Street,

Taoyuan 333, Taiwan

Tel $+8863328 \quad 1200$ ext 3846

Fax +8863328I200 ext 2667

Email yspeii@gmail.com

\begin{abstract}
Background/aims: Dual-task training may improve dual-task gait performance, balance, and cognition in older adults with and without cognitive impairment. Although music has been widely utilized in dementia management, there are no existing protocols for music-based dual-task training. This randomized controlled study developed a Musical Dual-Task Training (MDTT) protocol that patients with dementia can use to practice walking and making music simultaneously, to enhance attention control in patients during dual-tasking.
\end{abstract}

Methods: Twenty-eight adults diagnosed with mild-to-moderate dementia were assigned to the MDTT ( $\mathrm{n}=15)$ or control groups ( $\mathrm{n}=13)$. The MDTT group received MDTT, while the control group participated in non-musical cognitive and walking activities. The effects of MDTT were evaluated through the primary outcome of attention control, and secondary outcomes of dualtask performance, balance, falls efficacy, and agitation.

Results: The MDTT group showed a significant improvement in attention control, while the control group did not $(P<0.001)$. A significant effect favored MDTT over control treatment for the secondary outcome of falls efficacy $(P=0.02)$ and agitation $(P<0.01)$.

Conclusion: MDTT, a music therapy intervention that demands a high level of cognitive processing, enhances attention control, falls efficacy, and helps alleviate agitation in patients with mild-to-moderate dementia.

Keywords: music therapy, dementia, attention, agitation, incidental falls

\section{Introduction}

Dementia manifests as a decline in cognitive function, affecting memory, language, perception, and executive function. ${ }^{1}$ Recent studies have shown that the cognitive impairments associated with dementia are also reflected in changes in gait parameters such as decreased gait velocity and stability, ${ }^{2-4}$ contributing to increased incidences of falls in patients with dementia. ${ }^{5}$ Because patients with dementia are more prone to incidental falls, they have higher fracture rates than age-matched people with intact cognition..$^{6-8}$

Gait impairments are exacerbated by dual-task scenarios, for instance, walking and processing a cognitive task simultaneously. Compared with normal subjects, patients with dementia have been found to walk with a slower speed and higher stride time variability when asked to walk while counting backwards or naming as many words as possible starting with a specific letter. ${ }^{9-11}$ Dual-task gait impairments in persons with dementia may further increase their risk of falling, as functional mobility often requires individuals to walk while having a conversation. The difference in walking speed and stability between single-task and dual-task actions reflects the dual-task cost (DTC), which is a measurement of both cognitive impairment and the risk of falling. ${ }^{10,12}$ Performing a dual-task action requires effective and efficient allocation 
of attention between two tasks. ${ }^{13}$ The degree of difficulty when performing a dual-task action is related to the ability to dynamically shift attention between a physical and cognitive task. ${ }^{14,15}$ Given attention impairment, especially reflected under dual-task scenarios, is one of the earliest and most serious symptoms in people with dementia, ${ }^{16-18}$ there is a clear need for a fall prevention intervention that incorporates both cognitive and physical training. ${ }^{10,12}$

The effects of dual-task training have been evaluated in healthy older adults, ${ }^{19}$ older adults with balance impairments, ${ }^{20}$ older adults with a history of falls, ${ }^{21,22}$ patients with stroke, ${ }^{23}$ patients with Parkinson's disease, ${ }^{24}$ and patients with mild-to-moderate dementia. ${ }^{18,25-27}$ Among these studies, physical tasks included walking, throwing and catching balls, weight training, and balance training, while cognitive tasks included calculations, recalling numbers or words, and generating words according to semantic criteria. Following dual-task training, patients with dementia have been found to show improved frontal cognitive function, ${ }^{25,26}$ executive function, ${ }^{27}$ postural control during dual-task activities, ${ }^{26}$ balance, ${ }^{26,27}$ and reduced DTC under challenging dual-task conditions. ${ }^{18}$ However, no studies have examined the use of music therapy as a dual-task training protocol for patients with dementia or cognitive impairment, although musicbased multitask exercises have been found to improve gait and balance, and reduce incidences of falls in communitydwelling elderly individuals at risk of falling. ${ }^{28}$ Music therapy has been shown to have benefits on cognition in patients with dementia. ${ }^{29}$ Rhythmic auditory stimulation, a neurologic music therapy technique, has been applied to address the gait functions of patients with dementia. ${ }^{30}$ While the above-mentioned interventions attempted to address the cognitive and physical goals individually, a music therapy intervention that combines both the cognitive and physical training is warranted.

In the present study, we developed a Musical Dual-task Training (MDTT) program specifically designed for patients with dementia. Our MDTT program consists of dual-task physical and cognitive training structured around music under the direction of a music therapist. In our program, the patient may be asked to sing or play instruments while walking, and respond to cues from the therapist. Compared to regular dual-task training, MDTT may have additional benefits for patients with dementia. Music-making that involves great demands on attention and memory might elicit experience-dependent plasticity in the brain by strengthening the attention network. ${ }^{31-33}$ Individuals with musical training revealed greater activity levels in the areas of the brain controlling top-down attention such as the anterior cingulate, the frontal cortex, and the parietal cortex. ${ }^{34,35}$ Musicians' superior performance in many forms of visual and auditory attention, executive function tasks, task switching, and dual-task performance compared to non-musicians may be explained by this consistent and extensive recruitment of attention networks during music training. ${ }^{35-38}$ Furthermore, music-based dual-task training has been shown to integrate cognitive and physical components while increasing motivation and treatment adherence. ${ }^{28}$ The steady beat of music also facilitates rhythmic gait movements, as attentional resources may be released from the gait by synchronizing the gait with musical rhythm, making the gait more automatic. ${ }^{39}$ This facilitation may allow patients with dementia to perform dual-tasks more successfully.

Music is a unique tool in the care and rehabilitation of dementia, because, while impaired memory function is the most prominent symptom in patients with Alzheimer's disease (AD), many case reports have documented preferentially spared musical memory. ${ }^{40,41}$ The ability to play musical instruments remained intact after the onset of $\mathrm{AD}$, even though the patients might start to show difficulties in performing other daily activities. ${ }^{40}$ Playing a musical instrument is a procedural memory that engages brain areas including the basal ganglia and the cerebellum, which are less affected in early AD. ${ }^{42}$ As a music therapist, the primary investigator has witnessed numerous cases where patients with dementia continued to sing along with familiar and favorite tunes with accurate lyrics while their other long-term memories were impaired. Additionally, the primary investigator has also observed many patients with dementia who were more likely to recall and verbalize personal memories evoked by the music. The preservation of long-term musical memory may be explained by the research indicating the caudal anterior cingulate and the ventral presupplemental motor areas display relatively less atrophy in patients with $\mathrm{AD}$, and these areas are where the neural encoding of long-known, familiar music occurs. ${ }^{43,44}$ Another brain area that exhibits a relatively slower rate of cortical atrophy is the medial prefrontal cortex, ${ }^{45}$ which plays an important role in music-evoked autobiographical memory. ${ }^{46}$ The medial prefrontal cortex is involved in reward and emotions, and it activates during autobiographical memory retrieval. ${ }^{47}$ It also activates when listening to music that brings pleasure ${ }^{48}$ and during familiarity judgment about music. ${ }^{49}$ This relatively spared functioning of the medial prefrontal cortex may explain the ability of music to facilitate the recall of personal memory.

On the other hand, music also enhances memory in patients with AD ${ }^{50}$ Patients with $\mathrm{AD}$ were able to recognize 
more of the unfamiliar lyrics that were sung to them than spoken to them, ${ }^{50}$ suggesting a difference in the processing of musical and non-musical information. When musical information is processed and encoded, more brain areas are recruited in addition to those typically associated with the coding of general memory, such as the hippocampus and temporal lobe region. This results in a more robust and holistic encoding of musical memory that facilitates retrieval of information through an "alternative" or "backup" system that bypasses the brain areas affected by neurodegeneration. ${ }^{50}$

Music not only activates spared brain areas that are less affected by dementia pathology and may induce neuroplasticity after rehabilitation through its extensive engagement of a widespread network in the brain, but music also reduces anxiety and depression in patients with dementia through its impact on reward, arousal, and emotion networks. ${ }^{51,52}$ The relaxing effects of music may be explained by increased dopamine secretion and activation of the parasympathetic nervous system being induced by music..$^{53,54}$ Music-induced improvement of mood may further explain the enhanced performance of autobiographical memory in AD.

We investigated the effects of MDTT on attention control, dual-task performance, balance, falls efficacy, and agitation in older adults with mild-to-moderate dementia. We hypothesized that MDTT would improve attention control, our primary outcome measure, by practicing performing two tasks at the same time. We also hypothesized that, owing to the simultaneous emphasis on both cognitive and physical functioning, MDTT might enhance gait in dual-task conditions, promote balance, improve falls efficacy, and reduce agitation, our secondary outcome measures. This study is intended to motivate the development of cost-effective music therapy for executive dysfunction in patients with mild-tomoderate dementia.

\section{Method}

\section{Participants}

Participants were recruited via referrals by neurologists from a specialized dementia outpatient unit at a medical center and by case managers in cooperation with neurologists at a city district health center in Taiwan from October 2012 to March 2013. Participants were recruited if they 1) were aged 55 or older, 2) had mild-to-moderate dementia with a Clinical Dementia Rating (CDR) score of 0.5 , 1, or 2, as confirmed by a certified neurologist, and 3) were able to independently walk $10 \mathrm{~m}$ without using a walker, cane, or receiving assistance from another person. Participants were excluded if they 1) had other known neurological disorders, such as acute stroke or Parkinson's disease, or 2) had significant orthopedic, visual, or hearing impairments that hindered ambulation. The sample size was determined based on the work reported by Bruer et al, ${ }^{29}$ in which music therapy improved the Mini-Mental State Examination (MMSE) scores in immediate and next-day assessments in 17 elderly subjects with dementia.

\section{Interventionist}

The primary researcher was a board-certified music therapist who conducted the recruitment and assessment of the participants, and conducted the MDTT and control group sessions.

\section{Randomization and allocation}

An independent statistician randomized the placement of the participants into either the intervention or control group. The statistician used a 1:1 ratio according to a computergenerated list of random numbers. The participants were not stratified before randomization. Allocation assignment was concealed from the enrolling assessor. The intervention group received MDTT, and the control group participated in activities that involved non-musical cognitive tasks, such as reading newspapers and playing chess, as well as walking exercises. The activities in the control group were designed to provide participants with the same amount of time, attention, and contact as those in the MDTT group. Thus, we expected that both groups would experience a similar therapeutic relationship between the participants and therapist.

\section{Consent}

The Human Subjects Committee - Lawrence Campus (HSCL) at the University of Kansas and the Institutional Review Board of Chang Gung Medical Foundations, Taiwan, each approved this study. The methods were carried out in accordance with the Declaration of Helsinki. This study was registered at ClinicalTrials.gov (NCT01709188). At enrollment, interested and eligible participants or their guardians were asked to provide written informed consent before participating.

\section{Study design and setting}

In this randomized controlled, open-labeled, and parallelgroup study, participants attended training in eight weekly, individual, 60-min sessions. Each participant was scheduled to receive the intervention across a period of 2 months. An extended grace period of 3 weeks was provided to the participants if they missed up to three sessions throughout 
the regular 2-month experimental period. Individuals in both groups were assessed approximately 1 week before and 1 week after training to evaluate attention control, gait (walking speed and stride length under three task conditions), balance, falls efficacy, and agitation. The primary researcher was the assessor. The training for both the MDTT and control groups took place either at an activity room within the dementia specialty outpatient unit or at an activity room within the city district health center, according to where the participants were recruited. When transportation was an issue, sessions might be conducted at the participants' home.

\section{Intervention}

\section{The Musical Dual-task Training (MDTT) group}

The development of the MDTT protocol was modeled after the Transformational Design Model (TDM). ${ }^{55}$ TDM is a process that guides music therapists to translate research findings from neuroscience and behavioral science into designs for functional music therapy clinical applications. TDM includes six steps: Conducting an assessment, setting goals, designing a functional non-musical therapeutic exercise, translating the non-musical exercise into a musical exercise, reassessing, and transferring the therapeutic exercise to practice in functional daily living activities ${ }^{56}$ Following the steps of TDM, the primary investigator initially consulted literature to find a non-musical exercise that addresses the goal of attention control. Specifically, this non-musical exercise is dual-task training involving the performance of a physical task and a cognitive task at the same time. ${ }^{18,19,24,57}$ Both tasks require attention, such as walking and repeating names of animals or performing simple calculations. The primary investigator then translated this non-musical dualtask into a musical dual-task exercise, which involves the performance of a physical task and a musical task at the same time, such as walking while singing and walking while playing a simple percussive instrument. The non-musical dual task protocols described in the literature served as a template for developing the MDTT protocol. The scientific validity of this translation was established through the research findings, which indicated performing musical tasks demands attention, and individuals with musical training perform better on the attention control test than their counterparts without musical training, implying a potential experience-dependent neuroplasticity. ${ }^{58,59}$ MDTT was piloted in clinical settings with individuals with dementia in a nursing home. Feasibility and limited efficacy of the MDTT protocol were observed and discussed between the primary investigator and her music therapist mentor. The main focus of pilot testing was to determine the level of difficulties of MDTT combinations. No statistical data were collected during the pilot phase.

The MDTT protocol was designed to train participants in scenarios analogous to natural situations. The participants were asked to respond to obstacles (visual stimuli) and engage in conversation (auditory stimuli) while walking. The MDTT protocol included a musical task and a walking task. The musical task comprised two types of activities: singing and playing simple percussive musical instruments. Each of these activities had two versions with different attention levels. For the music task, the participants made music autonomously using their own self-generated rhythm in one version (whether playing a steady beat or playing freely) and received visual or auditory cues from the therapist in the other version (Table 1). In the walking task, the participants either walked forward or stepped sideways. Between the musical task and walking task, there were eight possible combinations of dual-task activities, ranging from singing familiar songs + walking (lowest cognitive load) to playing an instrument contingently + side-stepping (highest cognitive load). Thus, the therapist presented the participants with different challenges with varying cognitive loading to enhance the participant's ability. The participants were instructed to perform all eight combinations progressively within a session.

For instance, in the high-attention-demand version of the musical task, the participant sang while viewing a piece of paper with the lyrics to a song written in different colors. The participant was assigned a color and instructed to only sing lines written in the respective color, and not sing lines written in other colors. In another example of

Table I Level of attention demand of dual-tasks in MDTT

\begin{tabular}{|c|c|c|}
\hline Level of attention demand & Low & High \\
\hline \multicolumn{3}{|l|}{ Musical task } \\
\hline Singing & Singing familiar/preferred songs & Singing contingently on visual or auditory cues \\
\hline Instrument playing & $\begin{array}{l}\text { Playing steady beats on simple } \\
\text { percussive musical instruments }\end{array}$ & $\begin{array}{l}\text { Playing simple percussive musical instruments } \\
\text { contingently on visual or auditory cues }\end{array}$ \\
\hline Walking task & Forward walking at preferred pace & Stepping from side to side \\
\hline
\end{tabular}

Abbreviation: MDTT, Musical Dual-Task Training. 
this high-attention-demand version of the musical task, the participant was given a simple percussive instrument such as a tambourine or a maraca and instructed to only play continuously when he or she heard a particular section of a song. The music was selected according to the preferred songs of the individual participants, and included well-known folk songs and songs popular during the time period in which the participants were young adults. Individual music preferences were obtained from interviews with the participants and their guardians/caregivers. The music presented in the sessions was performed live by the music therapist who sang and played the guitar or keyboard. The live performance cued the patient to respond and provided temporal cues to facilitate walking movements.

\section{The control group}

The control group participated in activities involving 1) non-musical cognitive tasks and 2) walking exercises. The cognitive tasks were determined according to the preferences of the individual participants, and included chess, playing cards, reading, writing, completing mathematical exercises, puzzles, and games. During each control session, the music therapist alternately conducted cognitive tasks and took walks with the participant. Each participant took three-to-five walks across the room accompanied by the music therapist, depending on their physical condition that day. Between walks, participants sat and rested for 1-2 min.

\section{Primary outcome measurement: attention control}

We used the Trail Making Test Part A (TMT part A; Army Individual Test Battery, 1944) as a measure of attention control because this test measures executive function associated with visual searching, sequencing, and sustained attention. ${ }^{60}$ The Trail Making Test Part B was not included because our participants were unable to perform the test during the pilot testing. The participants drew lines to connect a set of 25 circles as quickly as possible, following the sequential order of numbers $(1,2,3 \ldots 25)$, while still maintaining accuracy. Performance on the TMT part A is quantified in terms of the number of seconds required to complete the task, such that lower scores indicate better performance. Theoretically, the threshold TMT part A score associated with an attention deficit is approximately $90 \mathrm{~s}$, and individuals are usually not asked to continue the test if they have not finished before this threshold. ${ }^{61,62}$ In the present study, we set the threshold at $600 \mathrm{~s}$ because our study included patients with moderate dementia who were expected to need extended time to complete the task. For those who could not finish the test in $600 \mathrm{~s}$ (gave up or could not continue due to limited attention or comprehension) or exceeded $600 \mathrm{~s}$ to finish, we recorded their time as $600 \mathrm{~s}$.

\section{Secondary outcome measurements Dual-task performance in gait analysis}

To assess the effect of DTC on spatial-temporal gait parameters, we performed gait analysis in three conditions, including the forward digit recall dual-task condition, backward digit recall dual-task condition, and single-task (control) condition. In the three conditions, the participants walked for $10 \mathrm{~m}$ at their preferred speed. We analyzed their gait using the FREE4ACT portable motion analysis system (Free4Act ${ }^{\circledR}$, LorAn-Engineering, Bologna, Italy), which yielded parameters of walking speed and stride length. The FREE4ACT obtained the gait data through a small, lightweight accelerometer attached to a belt placed at the L4-L5 inter-vertebral level. ${ }^{63}$

In the forward digit recall dual-task condition, the participants walked while verbally repeating sequences of three numbers read to them by the music therapist. For example, after the participants heard the numbers 3-6-7, they were required to say "three, six, seven". In the backward digit recall dual-task condition, the participants walked while verbally restating, in reverse order, sequences of two or three numbers. For example, after the participants heard numbers 3-6-7, they were required to say "seven, six, three". In the single-task condition, the participants simply walked without performing a verbal task.

The DTC denotes the ratio of change in performance between the single-task and dual-task conditions. The DTC of walking speed was calculated as follows:

$$
\text { DTC }=\frac{\begin{array}{l}
\text { Walking speed single-task }- \\
\text { Walking speed dual-task }
\end{array}}{\text { Walking speed single-task }} \times 100
$$

A DTC of 0 indicates that gait is not affected by a concurrent cognitive task, while a DTC of 100 represent the maximal influence a concurrent cognitive task could have on gait. We calculated the DTC of stride length and walking speed.

\section{Balance}

Balance was evaluated using the Timed Up and Go (TUG) test. The TUG measures the time required for a participant to rise and stand from a standard armchair, walk a distance 
of $3 \mathrm{~m}$, turn, walk back to the chair, and sit down. A higher amount of time taken to complete the task indicates higher balance impairment. ${ }^{64}$

\section{Falls efficacy}

Falls efficacy concerns the psychological aspects of falling. We assessed falls efficacy using the Falls Efficacy Scale International (FES-I). The participants reported how concerned they were about whether they would fall when doing activities such as getting dressed or undressed, taking a bath or shower, going up or down stairs, and going out to a social event. The FES-I has been used to evaluate fear of falling in geriatric populations, and the validity and sensitivity of this scale have also been established for individuals with cognitive impairments. Scoring is based on a 4-point scale, in which higher points indicate greater concern about falling. ${ }^{65}$

\section{Agitation}

We used the Chinese community-version Cohen-Mansfield Agitation Inventory scale (CMAI-C) to assess the frequency of agitated behaviors in the participants. The CMAI-C is a 29-item questionnaire rated by the caregivers to report the frequency of agitation over the most recent 2 weeks. ${ }^{66}$ Ratings of frequency are recorded on a 7-point scale, with scores ranging from 1, "never" to 7, "several times an hour". This instrument covers behaviors that are aggressive, physically nonaggressive, and verbally agitated, such as kicking, pushing, pacing and aimless wandering, repetitive questions, and screaming. ${ }^{67}$ The reliability and validity of this scale have been established in elderly people with dementia. ${ }^{68,69}$ Higher total points on the CMAI-C indicate a greater frequency of agitation in daily living.

\section{Data analysis}

We used SPSS version 22 to conduct statistical analyses following the intention-to-treat (ITT) principle. We first compared the MDTT and control groups in terms of pretraining characteristics using the Student's $t$-test for parametric data and the $\chi^{2}$ test for categorical data. Second, for each outcome measurement, we applied the method of Generalized Estimating Equations (GEEs) to examine whether the change between pretraining and posttraining assessments differed between the MDTT and control groups. Specifically, the GEE model included the pretraining score as an offset variable, and CDR as a factor in the equation. CDR was included in the model as GEE revealed that CDR was the major confounding variable that affected the baseline score and the effect of intervention. Finally, in cases of significant difference in the pair-wise comparison between the MDTT and control groups, one-tailed paired $t$-test was applied to examine the difference between pretraining and posttraining assessments for each group. Within each outcome measure, we applied a list-wise deletion in the case of missing data for the pre- or posttraining measurements. Reasons for missing data included participant failure to complete or attend the posttraining measurement meeting or a lack of information provided by the caregivers. The level of significance was $P<0.05$.

\section{Results}

\section{Participant characteristics}

Thirty-two patients diagnosed with dementia were screened for eligibility ( 21 from the dementia specialty outpatient unit, and 11 from the city district health center). Among these individuals, one declined to participate, and one was dropped because of an inability to complete the pre-training measurements. Thus, 30 participants were randomly allocated to the MDTT and control groups (CONSORT flowchart shown in Figure 1). After group allocation, one participant withdrew owing to a leg fracture that occurred before pretraining measurements, and a second participant withdrew owing to an objection to being placed in the control group, leaving 15 and 13 participants in the MDTT and control groups, respectively, who completed the posttraining measurements.

Table 2 shows the participant characteristics and pretraining measurements. The control group demonstrated significantly greater pretraining stride lengths in the forward digit recall dual-task $(P=0.03)$. We found no other significant differences in participant characteristics or pretraining measurements.

\section{Primary outcome measurement: attention control}

After the intervention, the change of TMT part A score was significantly higher in the MDTT group than in the control group $(P<0.001)$. Specifically, the TMT part A score decreased significantly in the MDTT group $(\mathrm{t}(1,14)=6.60$, $P=0.01)$, but not in the control group $(\mathrm{t}(1,12)=0.01, P=0.47)$ (Figure 2 and Table 3), indicating that attention improved in the MDTT group, but not in the control group.

\section{Secondary outcome measurements}

Gait under single-task and dual-task conditions

After the intervention, no significant difference in the change of walking speed was observed between the MDTT and control groups in any of the three conditions, 


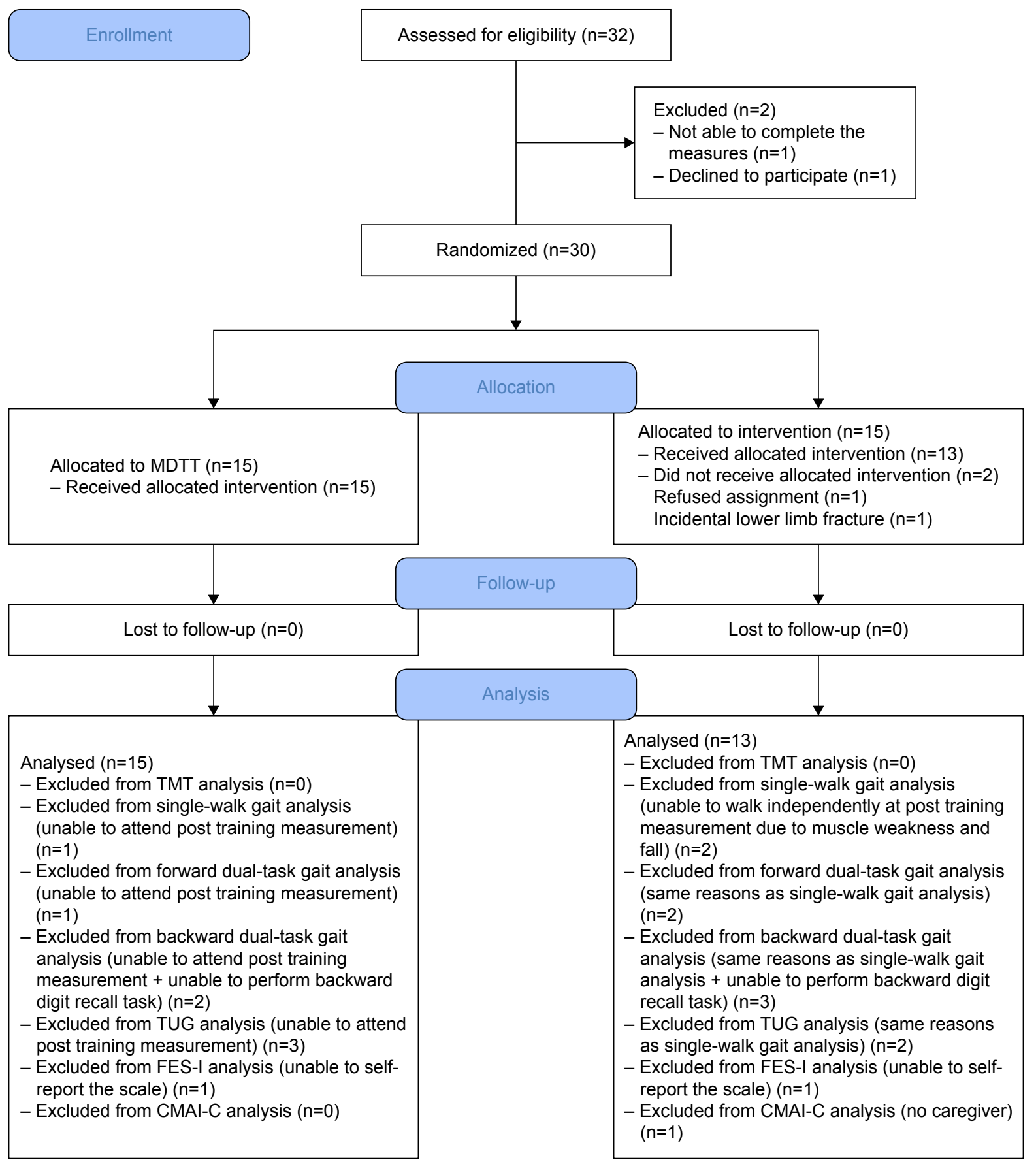

Figure I Flow chart of study pathway.

Abbreviations: MDTT, Musical Dual-Task Training; TMT, Trail Making Test; TUG, Timed Up and Go; FES-I, Falls Efficacy Scale International; CMAI-C, Cohen-Mansfield Agitation Inventory scale, Chinese community-version.

including the single-task, forward digit recall dual-task, and backward forward digit recall dual-task (all $P>0.05$ ). Likewise, the change of stride length did not differ significantly between the two groups in all three conditions (all $P>0.05$ ) (Table 4). These findings indicate that MDTT and control training had comparable effects on these gait parameters.

\section{DTC of walking speed and stride length}

After the intervention, no significant difference in the change of DTC walking speed was found between the MDTT and control groups in all three conditions, including the singletask, forward digit recall dual-task, and backward forward digit recall dual-task (all $P>0.05$ ). Analogously, the change of DTC of stride length did not differ between the two groups 
Table 2 Baseline characteristics and pretests of outcome measurements

\begin{tabular}{|c|c|c|c|}
\hline Characteristics & MDTT group & Control group & $P$-value \\
\hline Age (years) & $77.3 \pm 9.4$ & $77.3 \pm 10.0$ & 1.00 \\
\hline Gender, $\mathrm{n}$ & & & 0.26 \\
\hline Male & 6 & 8 & \\
\hline Female & 9 & 5 & \\
\hline MMSE & $16.4 \pm 7.3$ & $17.9 \pm 3.7$ & 0.53 \\
\hline CDR score, $\mathrm{n}$ & & & 0.29 \\
\hline 0.5 & 6 & 3 & \\
\hline I & 6 & 9 & \\
\hline 2 & 3 & I & \\
\hline Dementia type, $n$ & & & 0.77 \\
\hline$A D$ & 11 & 8 & \\
\hline $\mathrm{VaD}$ & 2 & 2 & \\
\hline Other & 2 & 3 & \\
\hline TMT part A (s) & $285.7 \pm 232.3$ & $267.8 \pm 173.9$ & 0.82 \\
\hline \multicolumn{4}{|c|}{ Walking speed (m/min) } \\
\hline Single & $57.4 \pm 8.6$ & $63.7 \pm 15.0$ & 0.20 \\
\hline Forward & $47.9 \pm 7.3$ & $53.2 \pm 16.7$ & 0.31 \\
\hline Backward & $45.4 \pm 6.0$ & $50.3 \pm 17.0$ & 0.36 \\
\hline \multicolumn{4}{|l|}{ Stride length (m) } \\
\hline Single & $I . I \pm 0.1$ & $1.2 \pm 0.2$ & 0.09 \\
\hline Forward & $1.0 \pm 0.1$ & $1.2 \pm 0.2$ & $0.03 *$ \\
\hline Backward & $1.0 \pm 0.1$ & $1.1 \pm 0.2$ & 0.08 \\
\hline \multicolumn{4}{|c|}{ Walking speed DTC (\%) } \\
\hline Forward & $15.7 \pm 13.6$ & $17.2 \pm 18.0$ & 0.81 \\
\hline Backward & $19.6 \pm 14.7$ & $21.8 \pm 21.7$ & 0.76 \\
\hline \multicolumn{4}{|c|}{ Stride length DTC (\%) } \\
\hline Forward & $10.0 \pm 11.4$ & $4.1 \pm 12.5$ & 0.22 \\
\hline Backward & $11.2 \pm 14.2$ & $9.5 \pm 13.5$ & 0.77 \\
\hline TUG (s) & $15.0 \pm 5.5$ & $17.2 \pm 16.6$ & 0.64 \\
\hline FES-I & $33.4 \pm 14.9$ & $23.7 \pm 10.3$ & 0.06 \\
\hline CMAI-C & $44.3 \pm 14.8$ & $34.7 \pm 10.8$ & 0.07 \\
\hline
\end{tabular}

Notes: Single $=$ Single-task walking only; Forward $=$ Forward digit recall dual-task; Backward = Backward digit recall dual-task; Data are presented as mean \pm SD. Independent $t$-test. $* P<0.05$.

Abbreviations: MDTT, Musical Dual-Task Training; MMSE, Mini-Mental State Examination; CDR, Clinical Dementia Rating; AD, Alzheimer's Disease; VaD, vascular dementia; TMT, Trail Making Test; DTC, dual-task cost; TUG, Timed Up and Go; FES-I, Falls Efficacy Scale International; CMAI-C, Cohen-Mansfield Agitation Inventory scale, Chinese community-version; SD, standard deviation.

in all three conditions (all $P>0.05$ ) (Table 4). These findings again indicate that MDTT was not superior to control training in terms of the effect on DTC.

\section{TUG performance}

There were no significant differences in the change of TUG between the MDTT and control groups (all $P>0.05$ ), indicating that the effect of MDTT and the effect of control training on balance were comparable (Table 4).

\section{Falls efficacy: FES-I score}

The MDTT group did not show deterioration of FES-I, as was observed in the control group $(P=0.02)$. Specifically, FES-I scores increased after control training $(\mathrm{t}(1,11)=4.03$,
$P=0.04$ ), indicating that participants in the control group expressed more severe concerns about falling after training. In contrast, the MDTT group had a stationary FES-I score $(\mathrm{t}(1,13)=0.13, P=0.45)$, indicating that MDTT maintained the level of concern about falling at a constant level (Table 5).

\section{Agitation: CMAI-C}

Compared to the control group, the MDTT group demonstrated a better control of agitation on CMAI-C $(P<0.01)$. Specifically, CMAI-C scores stayed unchanged after MDTT $(\mathrm{t}(1,14)=0.38, P=0.27)$, but increased after control training $(\mathrm{t}(1,11)=4.03, P=0.04)$, indicating that participants in the control group exhibited more frequent agitation after training. These findings suggest that, unlike control training, MDTT was able to prevent the escalation of agitation (Table 5).

\section{Harm}

We observed no adverse events such as falls or emotional distress in both the MDTT and control groups. The primary investigator was experienced in adjusting the pace and in reacting to the responses of the participants to alleviate potential distress induced by dual-tasking. In addition, many participants were not necessarily aware of their dual-task performance. They were not told whether or not they were doing anything "wrong". They simply continued practicing.

\section{Discussion}

We evaluated the effects of a MDTT program on individuals with mild-to-moderate dementia. We designed the MDTT with the hypothesis that it would improve the participants' cognitive and physical functions by enhancing participants' attention during dual-task conditions. Our primary results indicated that eight individual sessions of MDTT, each $60 \mathrm{~min}$ in length, led to a significant increase in attention control compared with the control intervention. To the best of our knowledge, this is the first study showing that dual-task music therapy can improve attention function in individuals with dementia.

Further, we tested our hypothesis that, given the augmented attentional performance, MDTT might increase walking speed and stride length during dual-tasking, thus reducing the DTC and facilitating balance. Our findings did not support this hypothesis. Note that the $P$-value of walking speed under the backward digit recall dual-task condition was 0.06 . If we had allowed a significance level of 0.06 , increasing the risk of a type I error, then this result would suggest that MDTT might enhance gait performance in patients with dementia under the condition with the highest attentional 

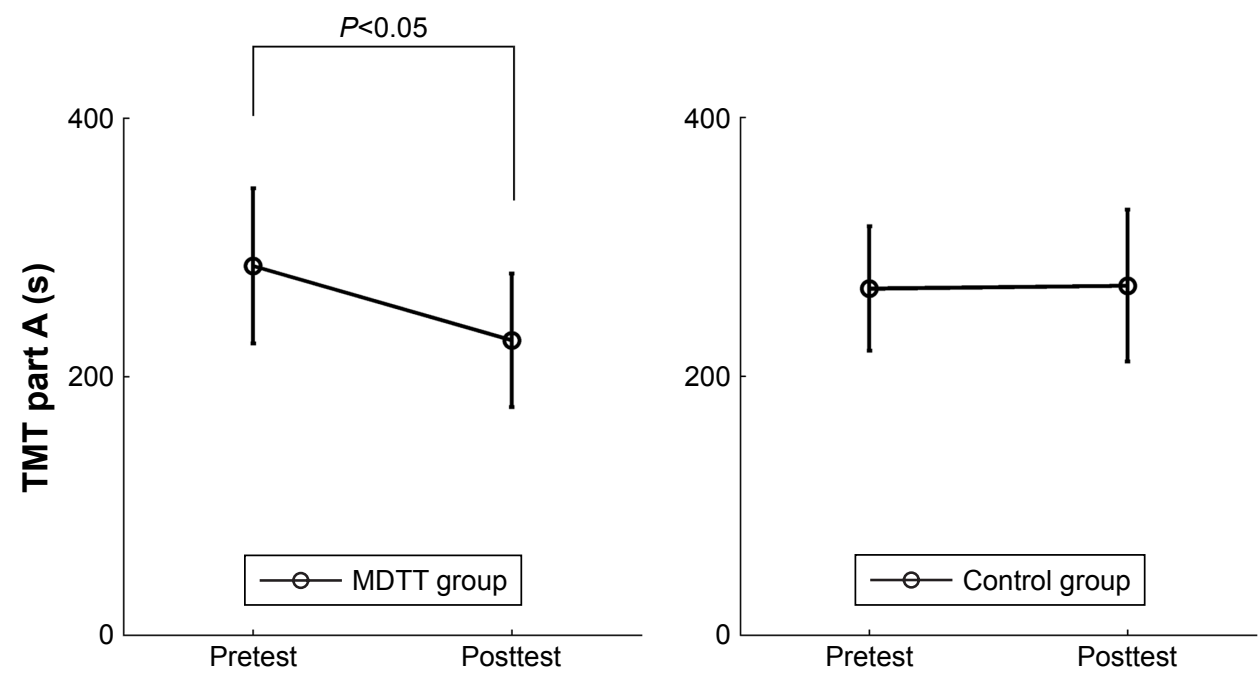

Figure 2 TMT part A for the MDTT and control groups at the pretest and posttest assessments. Abbreviations: TMT, Trail Making Test; MDTT, Musical Dual-Task Training.

demand. Interestingly, Schwenk et al ${ }^{18}$ also found that a dualtask exercise program reduced walking speed DTC while participants with mild-to-moderate dementia completed a complex cognitive task, while this was not the case for a less complex task. Their findings support the hypothesis that attention deficit becomes relevant when the attentional demands of a cognitive task exceed the capability of an individual.

Previous dual-task training studies found improved balance in healthy older adults and older adults with impaired balance via the TUG test. ${ }^{19,20}$ For individuals with AD, dualtask training also improved balance, although this improvement was only reflected by the Borg Balance Scale, and not the TUG test. ${ }^{27}$ Another study showed improved balance according to the TUG test. However, the researchers focused on the number of steps rather than the time taken to finish the TUG test, such that a comparison of finishing time produced no significant differences between the dual-task and control groups. ${ }^{26}$ This implies that the Borg Balance Scale is more sensitive to intervention-induced changes than the TUG test when assessing balance in individuals with dementia. Another possible explanation for the unchanged balance performance might be that some participants developed a habitual walking pattern in the present study. We observed that, when these individuals sensed a risk of falling (for instance, when making a turn during the TUG test), they would choose a slower walking speed. As a result, their performance in the TUG test was unaffected by either type of training.

In this study, we assessed self-rated fear of falling, because patients with dementia have a greater risk of falling, and this can restrict their daily activities. ${ }^{70} \mathrm{We}$ found that participants in the MDTT group gave similar ratings of falls efficacy before and after MDTT, whereas participants in the control group felt significantly more concerned about falling after the control treatment. Silsupadol et $\mathrm{al}^{20}$ suggested that patients receiving dual-tasking training might lose confidence regarding the performance of daily activities because these individuals are constantly challenged by increasingly difficult balance-keeping tasks. Thus, although MDTT did not reduce the fear of falling, this treatment is of clinical importance because individuals who completed MDTT did not exhibit an increased fear of falling. Agitation is an important issue for patients with dementia, and conventional music therapy has been shown to reduce agitated behaviors in these patients. ${ }^{71,72}$ MDTT maintained the same agitation levels after training, as before training, while the control activity elicited a significant increase in agitation.

Table 3 Changes in the primary outcome measurement, TMT part A, between the pretest and posttest assessments

\begin{tabular}{|c|c|c|c|c|c|c|c|c|c|}
\hline & \multicolumn{4}{|c|}{ MDTT group } & \multicolumn{4}{|c|}{ Control group } & \multirow[t]{2}{*}{$P$-value ${ }^{a}$} \\
\hline & $\mathbf{n}$ & Pretest & Posttest & Change $(95 \% \mathrm{Cl})$ & $\mathbf{n}$ & Pretest & Posttest & Change $(95 \% \mathrm{Cl})$ & \\
\hline $\begin{array}{l}\text { TMT } \\
\text { part A (s) }\end{array}$ & 15 & $285.7 \pm 232.3$ & $228.1 \pm 199.7$ & $-56.2(-102.5$ to 10.0$)$ & 13 & $267.8 \pm 173.9$ & $270.0 \pm 211.6$ & 2.2 (-67.7 to $7 \mid .2)$ & $<0.001 * * *$ \\
\hline
\end{tabular}

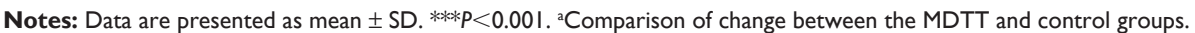

Abbreviations: TMT, Trail Making Test; MDTT, Musical Dual-Task Training; Cl, confidence interval; SD, standard deviation. 
Table 4 Changes in physical outcomes including walking, stride length, corresponding DTCs, and balance

\begin{tabular}{|c|c|c|c|c|c|c|c|c|c|}
\hline & \multicolumn{4}{|c|}{ MDTT group } & \multicolumn{4}{|c|}{ Control group } & \multirow[t]{2}{*}{$P$-value ${ }^{a}$} \\
\hline & $\mathbf{n}$ & Pretest & Posttest & Change $(95 \% \mathrm{Cl})$ & $\mathbf{n}$ & Pretest & Posttest & Change $(95 \% \mathrm{Cl})$ & \\
\hline \multicolumn{10}{|c|}{ Walking speed (m/min) } \\
\hline Single & 14 & $57.7 \pm 8.8$ & $60.3 \pm 16.8$ & $2.6(-6.1$ to 11.3$)$ & II & $67.6 \pm 12.7$ & $66.5 \pm 15.2$ & -1.1 (-6.4 to 4.2$)$ & 0.96 \\
\hline Forward & 14 & $48.2 \pm 7.5$ & $50.9 \pm 10.0$ & $2.7(-3.1$ to 8.5$)$ & 11 & $57.2 \pm 13.8$ & $57.6 \pm 13.6$ & $0.4(-8.2$ to 8.9$)$ & 0.42 \\
\hline Backward & 13 & $45.8 \pm 6.1$ & $49.9 \pm 8.6$ & 4.1 (-1.3 to 9.5$)$ & 10 & $55.3 \pm 11.7$ & $51.6 \pm 12.8$ & $-3.7(-10.2$ to 2.9$)$ & 0.06 \\
\hline \multicolumn{10}{|c|}{ Stride length (m) } \\
\hline Single & 14 & $\mathrm{I} . \mathrm{II} \pm 0.14$ & $1.16 \pm 0.27$ & $0.05(-0.09$ to 0.18$)$ & II & $1.26 \pm 0.18$ & $1.29 \pm 0.20$ & $0.02(-0.07$ to 0.12$)$ & 0.27 \\
\hline Forward & 14 & $1.01 \pm 0.15$ & $1.03 \pm 0.22$ & $0.02(-0.08$ to 0.12$)$ & II & $1.21 \pm 0.20$ & $1.18 \pm 0.24$ & $-0.03(-0.18$ to 0.12$)$ & 0.81 \\
\hline Backward & 13 & $1.00 \pm 0.15$ & $1.02 \pm 0.17$ & $0.03(-0.06$ to 0.11$)$ & 10 & $1.16 \pm 0.18$ & $1.13 \pm 0.24$ & $-0.02(-0.17$ to 0.12$)$ & 0.78 \\
\hline \multicolumn{10}{|c|}{ DTC on walking speed (\%) } \\
\hline Forward & 14 & $15.6 \pm 14.1$ & $13.6 \pm 13.7$ & $-2.0(-9.0$ to 5.0$)$ & 11 & $15.3 \pm 16.0$ & $12.4 \pm 14.8$ & $-2.9(-17.6$ to 11.9$)$ & 0.36 \\
\hline Backward & 13 & $19.2 \pm 15.2$ & $17.4 \pm 14.3$ & $-1.8(-13.1$ to 9.6$)$ & 10 & $17.6 \pm 16.0$ & $21.5 \pm 16.0$ & $3.9(-9.2$ to 16.9$)$ & 0.13 \\
\hline \multicolumn{10}{|c|}{ DTC on stride length (\%) } \\
\hline Forward & 13 & $9.8 \pm 11.8$ & $10.2 \pm 16.4$ & $0.4(-9.4$ to 10.3$)$ & II & $4.0 \pm 12.9$ & $8.1 \pm 14.8$ & $4.1(-7.8$ to 15.9$)$ & 0.14 \\
\hline Backward & 13 & $10.4 \pm 14.5$ & $12.1 \pm 12.8$ & $1.7(-7.9$ to II.3) & 10 & $9.1 \pm 14.3$ & $12.7 \pm 14.8$ & $3.6(-8.2$ to 15.5$)$ & 0.10 \\
\hline \multicolumn{10}{|l|}{ Balance } \\
\hline TUG (s) & 12 & $14.5 \pm 5.5$ & $15.5 \pm 6.6$ & $1.0(-0.3$ to 2.3$)$ & II & $12.5 \pm 4.1$ & $13.5 \pm 5.8$ & $1.0(-1.0$ to 2.9$)$ & 0.20 \\
\hline
\end{tabular}

Notes: Single = Single-task walking only; Forward = Forward digit recall dual-task; Backward = Backward digit recall dual-task. ${ }^{\mathrm{a} C o m p a r i s o n}$ of change between the MDTT and control groups.

Abbreviations: DTC, dual-task cost; MDTT, Musical Dual-Task Training; Cl, confidence interval; TUG, Timed Up and Go.

The potential specific link between the level of agitation and the increased attention control associated with MDTT needs to be further investigated in future studies.

The major limitation of this study was the small sample size and the initial differences between the MDTT and control groups, which resulted in low statistical power. Thus, our data include statistical information that could enable sample size estimation in future MDTT studies. Second, the interventionist was not blind to the data collection process, which might introduce a potential bias. The third limitation of this study was the lack of documentation regarding the history or incidence of falls during and after the intervention, although dual-task performance and falls efficacy are relevant to fall risk. Future work should include long-term follow-up assessments regarding the incidences of falls. Fourth, the control group's tasks were less complex. The control group's cognitive and physical tasks were delivered to participants separately, not in the dual task format. Thus, we could not rule out the possibility that the complexity of the tasks and the demands of dual-task also contributed to the differences in results in addition to music. Fifth, we did not record the qualitative data in terms of participants' individual performances during each session. We intended to ensure all participants received as similar as possible treatment, regardless of individual abilities, so all levels' tasks were introduced no matter how successfully the previous tasks were performed. It is beyond the scope of this study to report the individual differences in performing the individual tasks regarding the lengths and success rates, and this concern should be addressed in the future study.

Finally, since the MDTT applied in this study involved walking, our study was limited to participants who could maintain independent ambulation. Thus, future work may benefit from the inclusion of patients who are walkerdependent or wheelchair-bound.

This study recommends future studies with larger sample sizes investigate how MDTT benefits different types of dementia and other conditions with increased risk of falling

Table 5 Changes in falls efficacy and agitation

\begin{tabular}{|c|c|c|c|c|c|c|c|c|c|}
\hline & \multicolumn{4}{|c|}{ MDTT group } & \multicolumn{4}{|c|}{ Control group } & \multirow[t]{2}{*}{$P$-value ${ }^{a}$} \\
\hline & $\bar{n}$ & Pretest & Posttest & Change $(95 \% \mathrm{Cl})$ & $\bar{n}$ & Pretest & Posttest & Change $(95 \% \mathrm{Cl})$ & \\
\hline FES-I & 14 & $33.4 \pm 14.9$ & $33.1 \pm 12.4$ & $-0.3(-5.1$ to 4.5$)$ & 12 & $23.7 \pm 10.3$ & $26.6 \pm 14.3$ & $2.9(-0.3$ to 6.1$)$ & $0.02 *$ \\
\hline CMAI-C & 15 & $44.3 \pm 14.8$ & $41.9 \pm 13.4$ & $-2.4(-10.8$ to 6.0$)$ & 12 & $34.7 \pm 10.9$ & $39.0 \pm 15.7$ & $4.3(-0.4$ to 9.1$)$ & $<0.01 * *$ \\
\hline
\end{tabular}

Notes: Data are presented at mean \pm SD. ${ }^{* P}<0.05 ; * * P<0.01$. ${ }^{2}$ Comparison of change between the MDTT and control groups. Higher scores indicate a greater concern about falling and a greater frequency of agitation in daily living.

Abbreviations: MDTT, Musical Dual-Task Training; Cl, confidence interval; FES-I, Falls Efficacy Scale International; CMAI-C, Cohen-Mansfield Agitation Inventory scale, Chinese community-version; SD, standard deviation. 
that are related to an executive function decline. It would also be intriguing to conduct a longitudinal study that evaluates the long-term effects of MDTT on the rate of cognitive decline.

This study intends to establish evidence for MDTT that could be applied by credentialed music therapists who have training in music and science, have accomplished a clinical internship, and passed a board exam or equivalent according to the regulation of a given country. Other professionals or caregivers have the potential to perform MDTT with potential challenges in execution. The challenges they face may include, but are not limited to, a lack of knowledge of songs, the demand in playing accompaniment instruments while giving extra cues, and the adaption of dual-task on the spot, to not only motivate but also alleviate the potential agitation the participants may exhibit. For non-music therapists attempting MDTT, we recommend a co-treatment with a credentialed music therapist while conducting MDTT, or conducting MDTT under the consultation of a credentialed music therapist. The idea of increasing the availability of MDTT by having non-music therapists perform it is promising, but the feasibility and efficacy of MDTT when carried out by nonmusic therapists are yet to be discovered and will need to be evaluated in future studies.

\section{Conclusion}

This study is the first to support the feasibility and efficacy of a music-based dual-task training protocol, MDTT, for patients with mild-to-moderate dementia. It is also the first to highlight the cognitive, rather than the physical aspects of a dual-task training program, which has a primary effect of improving attention control. While most studies have attributed the results of dual-task activities to improved physical fitness, the effects of our dual-task MDTT protocol were more closely associated with improved attention, falls efficacy, and agitation. This study provides conceptual and clinical insights for researchers and clinicians developing music therapy techniques for patients with mild-to-moderate dementia.

\section{Acknowledgments}

This work was supported by the Ministry of Education, Taiwan (grant numbers EMRPD 1C0291, 1B0341, and 1A0861). The authors wish to thank all the participants in this study, Dr Wen-Chuin Hsu and colleagues in Dementia Center, Chang Gung Memorial Hospital at Taoyuan, Dr Jean Chou, the former director of the Songshan District Health Center of Taipei City, for assistance with participants recruitment, and Dr Alicia Clair, Professor Emeritus at the University of Kansas, for her guidance throughout the study.

\section{Author contributions}

YLC initiated, designed, and performed the study, analyzed the results, drafted, and revised the manuscript. YCP analyzed the results and was involved in the research design and execution, and revised the manuscript. All authors contributed toward data analysis, revising the paper and agree to be accountable for all aspects of the work.

\section{Disclosure}

The authors report no conflicts of interest in this work.

\section{References}

1. Encyclopedia ADAMM. Dementia. http://www.ncbi.nlm.nih.gov/ pubmedhealth/PMH0001748/. Accessed September 26, 2011.

2. Beauchet O, Allali G, Thiery S, Gautier J, Fantino B, Annweiler C. Association between high variability of gait speed and mild cognitive impairment: a cross-sectional pilot study. J Am Geriatr Soc. 2011; 59(10):1973-1974.

3. Scherder E, Eggermont L, Swaab D, et al. Gait in ageing and associated dementias; its relationship with cognition. Neuroscience and Biobehavioral Reviews. 2007;31(4):485-497.

4. Waite LM, Grayson DA, Piguet O, Creasey H, Bennett HP, Broe GA. Gait slowing as a predictor of incident dementia: 6-year longitudinal data from the Sydney Older Persons Study. J Neurol Sci. 2005;229-230: 89-93

5. Webster KE, Merory JR, Wittwer JE. Gait variability in community dwelling adults with Alzheimer disease. Alzheimer Dis Assoc Disord. 2006;20(1):37-40.

6. Allan LM, Ballard CG, Rowan EN, Kenny RA. Incidence and prediction of falls in dementia: a prospective study in older people. PLoS One. 2009;4(5):e5521.

7. Buchner DM, Larson EB. Falls and fractures in patients with Alzheimertype dementia. JAMA. 1987;257(11):1492-1495.

8. Morris JC, Rubin EH, Morris EJ, Mandel SA. Senile dementia of the Alzheimer's type: an important risk factor for serious falls. J Gerontol. 1987;42(4):412-417.

9. Allali G, Assal F, Kressig RW, Dubost V, Herrmann FR, Beauchet O Impact of impaired executive function on gait stability. Dement Geriatr Cogn Disord. 2008;26(4):364-369.

10. Ijmker T, Lamoth CJ. Gait and cognition: the relationship between gait stability and variability with executive function in persons with and without dementia. Gait Posture. 2012;35(1):126-130.

11. Lamoth CJ, van Deudekom FJ, van Campen JP, Appels BA, de Vries OJ, Pijnappels M. Gait stability and variability measures show effects of impaired cognition and dual tasking in frail people. J Neuroeng Rehabil. 2011;8:2.

12. Sheridan PL, Hausdorff JM. The role of higher-level cognitive function in gait: executive dysfunction contributes to fall risk in Alzheimer's disease. Dement Geriatr Cogn Disord. 2007;24(2):125-137.

13. Sheridan PL, Solomont J, Kowall N, Hausdorff JM. Influence of executive function on locomotor function: divided attention increases gait variability in Alzheimer's disease. J Am Geriatr Soc. 2003;51(11): 1633-1637.

14. Hawkes TD, Siu KC, Silsupadol P, Woollacott MH. Why does older adults' balance become less stable when walking and performing a secondary task? Examination of attentional switching abilities. Gait Posture. 2012;35(1):159-163. 
15. Siu KC, Chou LS, Mayr U, Donkelaar P, Woollacott MH. Does inability to allocate attention contribute to balance constraints during gait in older adults? J Gerontol A Biol Sci Med Sci. 2008;63(12):1364-1369.

16. Baddeley AD, Baddeley HA, Bucks RS, Wilcock GK. Attentional control in Alzheimer's disease. Brain. 2001;124(Pt 8):1492-1508.

17. Perry RJ, Hodges JR. Attention and executive deficits in Alzheimer's disease. A critical review. Brain. 1999;122(Pt 3):383-404.

18. Schwenk M, Zieschang T, Oster P, Hauer K. Dual-task performances can be improved in patients with dementia: a randomized controlled trial. Neurology. 2010;74(24):1961-1968.

19. Plummer-D'Amato P, Cohen Z, Daee NA, Lawson SE, Lizotte MR, Padilla A. Effects of once weekly dual-task training in older adults: a pilot randomized controlled trial. Geriatr Gerontol Int. 2012;12(4): 622-629.

20. Silsupadol P, Shumway-Cook A, Lugade V, et al. Effects of singletask versus dual-task training on balance performance in older adults: a double-blind, randomized controlled trial. Arch Phys Med Rehabil. 2009;90(3):381-387.

21. Dorfman M, Herman T, Brozgol M, et al. Dual-task training on a treadmill to improve gait and cognitive function in elderly idiopathic fallers. J Neurol Phys Ther. 2014;38(4):246-253.

22. You JH, Shetty A, Jones T, Shields K, Belay Y, Brown D. Effects of dual-task cognitive-gait intervention on memory and gait dynamics in older adults with a history of falls: a preliminary investigation. NeuroRehabilitation. 2009;24(2):193-198.

23. Plummer-D'Amato P, Kyvelidou A, Sternad D, Najafi B, Villalobos RM, Zurakowski D. Training dual-task walking in community-dwelling adults within 1 year of stroke: a protocol for a single-blind randomized controlled trial. BMC Neurol. 2012;12:129.

24. Yogev-Seligmann G, Giladi N, Brozgol M, Hausdorff JM. A training program to improve gait while dual tasking in patients with Parkinson's disease: a pilot study. Arch Phys Med Rehabil. 2012;93(1):176-181.

25. Coelho FG, Andrade LP, Pedroso RV, et al. Multimodal exercise intervention improves frontal cognitive functions and gait in Alzheimer's disease: a controlled trial. Geriatr Gerontol Int. 2013;13(1):198-203.

26. de Andrade LP, Gobbi LT, Coelho FG, Christofoletti G, Costa JL, Stella F. Benefits of multimodal exercise intervention for postural control and frontal cognitive functions in individuals with Alzheimer's disease: a controlled trial. J Am Geriatr Soc. 2013;61(11):1919-1926.

27. Pedroso RV, Coelho FG, Santos-Galduroz RF, Costa JL, Gobbi S, Stella F. Balance, executive functions and falls in elderly with Alzheimer's disease (AD): a longitudinal study. Arc Gerontol Geriatr. 2012;54(2):348-351.

28. Trombetti A, Hars M, Herrmann FR, Kressig RW, Ferrari S, Rizzoli R. Effect of music-based multitask training on gait, balance, and fall risk in elderly people: a randomized controlled trial. Arch Intern Med. 2011; 171(6):525-533.

29. Bruer RA, Spitznagel E, Cloninger CR. The temporal limits of cognitive change from music therapy in elderly persons with dementia or dementia-like cognitive impairment: a randomized controlled trial. J Music Ther. 2007;44(4):308-328.

30. Clair AA, O'Konski M. The effect of rhythmic auditory stimulation (RAS) on gait characteristics of cadence, velocity, and stride length in persons with late stage dementia. J Music Ther. 2006;43(2):154-163.

31. Bugos JA, Perlstein WM, McCrae CS, Brophy TS, Bedenbaugh PH. Individualized piano instruction enhances executive functioning and working memory in older adults. Aging Ment Health. 2007;11(4): 464-471.

32. Wan CY, Schlaug G. Music making as a tool for promoting brain plasticity across the life span. Neuroscientist. 2010;16(5):566-577.

33. Lappe C, Trainor LJ, Herholz SC, Pantev C. Cortical plasticity induced by short-term multimodal musical rhythm training. PLoS One. 2011;6(6):e21493.

34. Pallesen KJ, Brattico E, Bailey CJ, et al. Cognitive control in auditory working memory is enhanced in musicians. PLoS One. 2010;5(6): e11120.
35. Strait DL, Kraus N, Parbery-Clark A, Ashley R. Musical experience shapes top-down auditory mechanisms: evidence from masking and auditory attention performance. Hear Res. 2010;261(1-2):22-29.

36. Bialystok E, Depape AM. Musical expertise, bilingualism, and executive functioning. J Exp Psychol Hum Percept Perform. 2009;35(2): 565-574.

37. Moradzadeh L, Blumenthal G, Wiseheart M. Musical training, bilingualism, and executive function: a closer look at task switching and dual-task performance. Cogn Sci. 2015;39(5):992-1020.

38. Rodrigues AC, Loureiro MA, Caramelli P. Long-term musical training may improve different forms of visual attention ability. Brain Cogn. 2013;82(3):229-235.

39. Maclean LM, Brown LJ, Astell AJ. The effect of rhythmic musical training on healthy older adults' gait and cognitive function. Gerontologist. 2014;54(4):624-633.

40. Baird A, Samson S. Memory for music in Alzheimer's disease: unforgettable? Neuropsychol Rev. 2009;19(1):85-101.

41. Cuddy LL, Duffin J. Music, memory, and Alzheimer's disease: is music recognition spared in dementia, and how can it be assessed? Med Hypotheses. 2005;64(2):229-235.

42. Hirono N, Mori E, Ikejiri Y, et al. Procedural memory in patients with mild Alzheimer's disease. Dement Geriatr Cogn Disord. 1997;8(4): 210-216.

43. Clark CN, Warren JD. Music, memory and mechanisms in Alzheimer's disease. Brain. 2015;138(Pt 8):2122-2125.

44. Jacobsen JH, Stelzer J, Fritz TH, Chetelat G, La Joie R, Turner R. Why musical memory can be preserved in advanced Alzheimer's disease. Brain. 2015;138(Pt 8):2438-2450.

45. Thompson PM, Hayashi KM, de Zubicaray G, et al. Dynamics of gray matter loss in Alzheimer's disease. J Neurosci. 2003;23(3):994-1005.

46. Janata P. The neural architecture of music-evoked autobiographical memories. Cereb Cortex. 2009;19(11):2579-2594.

47. Gilboa A. Autobiographical and episodic memory - one and the same? Evidence from prefrontal activation in neuroimaging studies. Neuropsychologia. 2004;42(10):1336-1349.

48. Blood AJ, Zatorre RJ. Intensely pleasurable responses to music correlate with activity in brain regions implicated in reward and emotion. Proc Natl Acad Sci U S A. 2001;98(20):11818-11823.

49. Platel H, Baron JC, Desgranges B, Bernard F, Eustache F. Semantic and episodic memory of music are subserved by distinct neural networks. Neuroimage. 2003;20(1):244-256.

50. Simmons-Stern NR, Budson AE, Ally BA. Music as a memory enhancer in patients with Alzheimer's disease. Neuropsychologia. 2010;48(10): 3164-3167.

51. Sarkamo T. Music for the ageing brain: Cognitive, emotional, social, and neural benefits of musical leisure activities in stroke and dementia. Dementia (London). Epub 2017 Jan 01.

52. Sihvonen AJ, Sarkamo T, Leo V, Tervaniemi M, Altenmuller E, Soinila S. Music-based interventions in neurological rehabilitation. Lancet Neurol. 2017;16(8):648-660.

53. Okada K, Kurita A, Takase B, et al. Effects of music therapy on autonomic nervous system activity, incidence of heart failure events, and plasma cytokine and catecholamine levels in elderly patients with cerebrovascular disease and dementia. Int Heart J. 2009;50(1):95-110.

54. Salimpoor VN, Benovoy M, Larcher K, Dagher A, Zatorre RJ. Anatomically distinct dopamine release during anticipation and experience of peak emotion to music. Nat Neurosci. 2011;14(2):257-262.

55. Thaut MH. Rhythm, music, and the brain: Scientific foundations and clinical applications. Vol 7. New York: Routledge; 2005.

56. Thaut MH, Hoemberg V. Handbook of neurologic music therapy. Oxford: Oxford University Press; 2014

57. Plummer P, Villalobos RM, Vayda MS, Moser M, Johnson E. Feasibility of dual-task gait training for community-dwelling adults after stroke: a case series. Stroke Res Treat. 2014;2014:538602.

58. Patston LL, Hogg SL, Tippett LJ. Attention in musicians is more bilateral than in non-musicians. Laterality. 2007;12(3):262-272. 
59. Strait DL, Kraus N. Can you hear me now? Musical training shapes functional brain networks for selective auditory attention and hearing speech in noise. Front Psychol. 2011;2:113.

60. Rabin LA, Barr WB, Burton LA. Assessment practices of clinical neuropsychologists in the United States and Canada: a survey of INS, NAN, and APA Division 40 members. Arch Clin Neuropsychol. 2005;20(1): 33-65.

61. AshendorfL, Jefferson AL, O'ConnorMK, ChaissonC, Green RC, SternRA. Trail Making Test errors in normal aging, mild cognitive impairment, and dementia. Arch Clin Neuropsychol. 2008;23(2):129-137.

62. Baity MR. Brief rating scales for the assessment of cognitive and neuropsychological status. In: Baer L, Blais MA, editors. Handbook of Clinical Rating Scales and Assessment in Psychiatry and Mental Health. Totowa, NJ: Humana Press; 2010:252.

63. Maggio M, Ceda GP, Ticinesi A, et al. Instrumental and non-instrumental evaluation of 4-meter walking speed in older individuals. PLoS One. 2016;11(4):e0153583.

64. Christofoletti G, Oliani MM, Gobbi S, Stella F, Bucken Gobbi LT, Renato Canineu P. A controlled clinical trial on the effects of motor intervention on balance and cognition in institutionalized elderly patients with dementia. Clin Rehab. 2008;22(7):618-626.

65. Kempen GI, Yardley L, van Haastregt JC, et al. The Short FES-I: a shortened version of the falls efficacy scale-international to assess fear of falling. Age Ageing. 2008;37(1):45-50.
66. Lai CKY. The use of the Cohen-Mansfield Agitation Inventory in the assessment of agitation in people with dementia: applicability in Hong Kong. HK J Gerontol. 2002;14(1\&2):66-69.

67. Cohen-Mansfield J, Werner P, Watson V, Pasis S. Agitation among elderly persons at adult day-care centers: the experiences of relatives and staff members. Int Psychogeriatr. 1995;7(3):447-458.

68. Lai JY. The Cohen-Mansfield Agitation Inventory: development of a Chinese version. Chin J Nurs. 2010;45(6):500-504.

69. Choy CN, Lam LC, Chan WC, Li SW, Chiu HF. Agitation in Chinese elderly: validation of the Chinese version of the Cohen-Mansfield Agitation Inventory. Int Psychogeriatr. 2001;13(3):325-335.

70. Hauer KA, Kempen GI, Schwenk M, et al. Validity and sensitivity to change of the falls efficacy scales international to assess fear of falling in older adults with and without cognitive impairment. Gerontology. 2011;57(5):462-472.

71. Ledger AJ, Baker FA. An investigation of long-term effects of group music therapy on agitation levels of people with Alzheimer's Disease. Aging Ment Health. 2007;11(3):330-338.

72. Ridder HM, Stige B, Qvale LG, Gold C. Individual music therapy for agitation in dementia: an exploratory randomized controlled trial. Aging Ment Health. 2013;17(6):667-678.
Neuropsychiatric Disease and Treatment

\section{Publish your work in this journal}

Neuropsychiatric Disease and Treatment is an international, peerreviewed journal of clinical therapeutics and pharmacology focusing on concise rapid reporting of clinical or pre-clinical studies on a range of neuropsychiatric and neurological disorders. This journal is indexed on PubMed Central, the 'PsycINFO' database and CAS,

\section{Dovepress}

and is the official journal of The International Neuropsychiatric Association (INA). The manuscript management system is completely online and includes a very quick and fair peer-review system, which is all easy to use. Visit http://www.dovepress.com/testimonials.php to read real quotes from published authors. 